\title{
Calculation of minimum crack size for growth under RCF between wheel and rail
}

\author{
H. K. Jun ${ }^{1}$, D. I. Fletcher ${ }^{2}$, H. S. Jung ${ }^{1}$, G. H. Lee ${ }^{1}$ \& D. H. Lee ${ }^{1}$ \\ ${ }^{1}$ Korea Railroad Research Institute, Rep. of Korea \\ ${ }^{2}$ Department of Mechanical Engineering, Sheffield University, UK
}

\begin{abstract}
Surface initiated cracks in rails by repeated rolling contact between wheels and rails have been one of the growing problems in high speed rail. Estimation of a minimum crack size for growth and its growth rate are the most demanding work to develop a cost effective rail maintenance strategy and prevent rails from failure. In this study, we did a series of modelling runs to predict a minimum crack size for growth, which was defined as a crack that grew fast enough to 'keep ahead' of its removal by surface truncation in various contact conditions. For this purpose, we developed crack growth simulation software based on the F\&B model suggested by Fletcher and Beynon, and calculated the minimum crack sizes for growth considering the effect of dominant contact contributors such as contact pressure, surface friction coefficient, surface wear rate and initial defect size. The minimum crack sizes for growth were calculated from 0.19 to $3.60 \mathrm{~mm}$.
\end{abstract}

Keywords: rolling contact fatigue, surface crack, fatigue crack growth, wheel/rail contact.

\section{Introduction}

Rolling contact fatigue (RCF) $[1,2]$ is a damage phenomenon that appears on the surface and subsurface of rails. This phenomenon is the result of repeated overstressing on the surface material by the millions of intense wheel and rail contacts. Surface initiated cracks, like a squat [3], is a well-known defect that is observed on the running band of tangent rails. Once a crack has initiated and grown over a certain size, without proper maintenance, it will continually grow and finally bring a whole rail breakage. Crack growth rate at which it progresses 
into the surface is influenced by tens of contact contributors and it is not easy to control them to reduce the damage. Instead, the periodic removal of the damaged surface layer is adapted in the field. Removal of surface material eliminates the damaged layer which brings a surface crack initiation, and also truncates existing cracks too. The optimal situation is to continuously remove just the right amount of metal to control surface crack initiation and propagation of short cracks when the growth rate is still low [4]. If a short crack does not have enough growth rate then it will not grow into a long crack, and it may eventually disappear owing to surface truncation. This means that there is a Minimum Crack Size for Growth (MCSG). In other words, a crack can be considered as a real crack only when it is larger than the MCSG. Nevertheless, such a size has not been clearly determined yet because there are too many contributors which are not easy to quantify. To determine the MCSG, it is necessary to calculate the exact moment when the crack starts continued growth by considering the crack advance rate and the crack truncation rate. Fracture mechanics is concerned with providing a method [1, -2] for predicting the failure of a material by crack growth analysis. A great deal of research [5-15] has been made to predict crack growth during wheels rolling over a crack, and great advances have been achieved. But the calculation of MCSG has not been fully investigated yet.

\section{RCF crack growth}

\subsection{Rail crack management}

Fig. 1 shows the schematics of maintenance action strategy considering a crack growth to ensure the safety of rails. Normally, cracks will not be detected until they have grown to a certain size. But once a crack is detected, the track engineer has to decide if it is a current risk that requires immediate removal or will become a risk in the future. But the time span from detection to failure is not always given enough for the maintenance of defected rail.

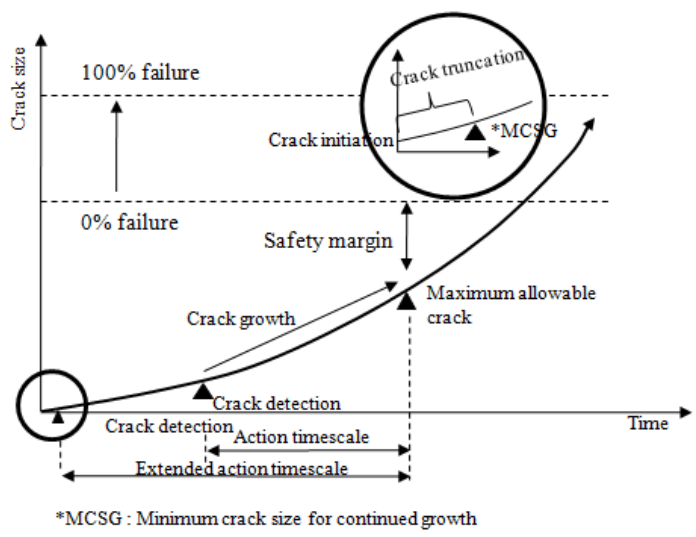

Figure 1: $\quad$ Schematics of crack growth and its effect on action time scale. 
Therefore it is necessary to extend the action time scale by predicting the minimum crack size when it starts to continued to grow to get more chances for maintenance.

\subsection{Stress intensity factor method}

The Green's function method was originally developed to apply results from a stress intensity factor (SIF) calculation to a wide range of input conditions. It treated an arbitrary crack face load as a series of point forces and to sum up the combined SIF for the entire arbitrary load. The Green's functions for calculating the SIF about an inclined edge crack, when a normal or tangential point load was applied at surface of half space, was developed by Rooke et al. [11]. He calculated the stresses along the line of a crack and integrated them along the crack. The method extended to apply rolling contact problem by Fletcher and Beynon [13]. They developed a simple " $2 \mathrm{D}$ " method for the calculation of SIF for an inclined edge surface crack under Hertzian contact and crack face friction loading by implementing the crack line Green's function. And it was further extended to an inclined semi-circular and semi-elliptical crack under a 3D semicircular and semi-elliptical contact patch $[14,15]$.

Although the finite element method [5-10] is very comprehensive, it has the major drawback that its complexity makes model generation very timeconsuming, especially a three dimensional crack under the elliptical shape contact load, and solving the models is slower than any other alternative approaches. While the Green function method does not have wide applicability compare to the FEM, it gives fast and reasonable accuracy. Crack growth analysis requires millions of repeated cycle, so fast calculation is critical. By this reason, we determined the Green's function method as an appropriate method for the crack growth analysis.

\subsection{MCSG calculation}

To calculate the MCSG, following assumptions were implemented through all the studies for the simplicity of the calculation. These assumptions can bring a little higher crack growth than physical conditions, because the assumptions are conservative. But the assumptions seemed quite adequate at this moment for understanding complicated rolling contact fatigue crack growth phenomena quantitatively.

(1)The contact between wheel and rail was assumed as it followed the Hertzian contact.

(2) Although, the wear rate would be varied by the contact and surface condition, but for the simplicity of analysis, it was regarded as constant value as $0.5,1.0$ and $1.5 \mathrm{~nm} /$ cycle.

(3) The initial crack aspect ratio a/b was assumed to be maintained as constant during the simulation.

(4) The stress intensity factor for a wide slot crack was calculated with the stress intensity factor handbook, developed by Murakami [19], and it was converted 
to calculate those for a semi-circular crack and a semi-elliptical crack by using a geometry factor for simplicity of calculation.

(5) We considered only the phase II [2] crack growth driven by the contact stress between wheel and rail. Also only linear elastic fracture mechanics was applied to calculate crack growth even though a very short crack.

(6) We assumed that all the wheels passed right above the center line of crack and they produced the maximum contact stress and this assumption accelerated the crack growth rate.

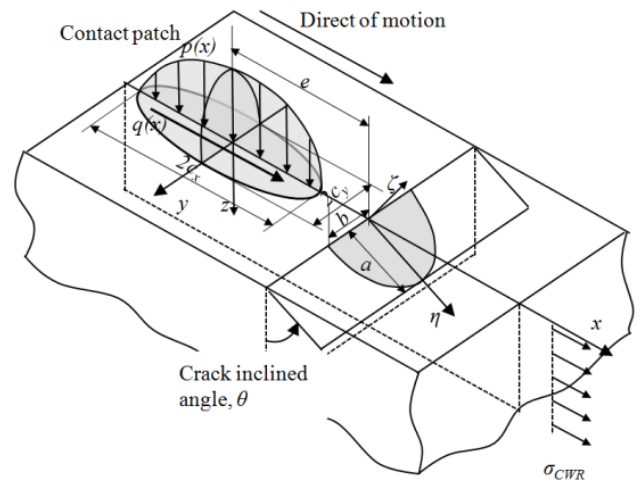

Figure 2: Schematic representation of an inclined edge crack in a half-space under Hertzian contact and thermal stress loading [14].

Fig. 3 is the process to calculate the MCSG. Two cases were studied to compare the effect of periodic grinding. Fig. 3(a) is the process which does not consider the periodic grinding maintenance. Variable initial crack sizes are assumed to start simulation and the calculations are repeated until the growing crack is longer than the initial crack $\left(\mathrm{a}_{\mathrm{i}}>\mathrm{a}_{0}\right)$. The initial crack growth owing to the

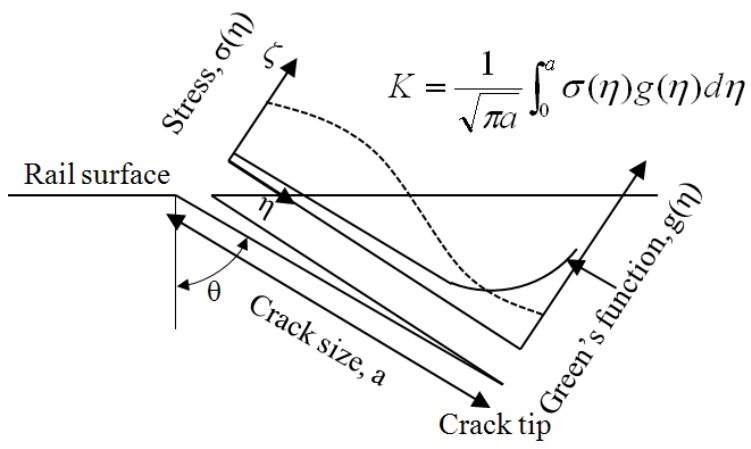

Figure 3: Schematic representation of SIF calculation by combining the stress function along the line of crack and Green's function [11]. 
contact is simulated by increasing crack size at crack tip $\left(a_{i}+=d a_{c}\right)$. While the wearing is simulated by truncating crack size at crack mouth $\left(a_{i}-=d_{w}\right)$. Fig. $3(b)$ is the process which does consider the periodic grinding maintenance. The periodic grinding is also simulated by truncating the crack mouth every time when the accumulated gross tons reaches the grinding period $\left(a_{i}-=d a_{g}\right)$. After the end of a cycle, if the remaining crack size is longer than the initial crack size $\left(\mathrm{a}_{\mathrm{i}}>\mathrm{a}_{0}\right)$ then it means that the crack will continuously grow. We therefore determine the initial crack size as the MCSG. While, if the remaining crack size is shorter than the initial crack size then it means that the growing crack will be worn out after all. In this case, we increase the initial crack size as $1 \%$ of its size and re-performed the crack growth analysis. More accurate crack size can be obtained if we reduce the increment, but it requires a huge amount of simulation time. These processes were developed as computer software to utilize the crack growth simulation because they required millions of repeated cycles. To verify the software, we calculated the SIFs of $45^{\circ}$ inclined circular cracks case and compared the results with well-known solutions [14] and got a good correlation.

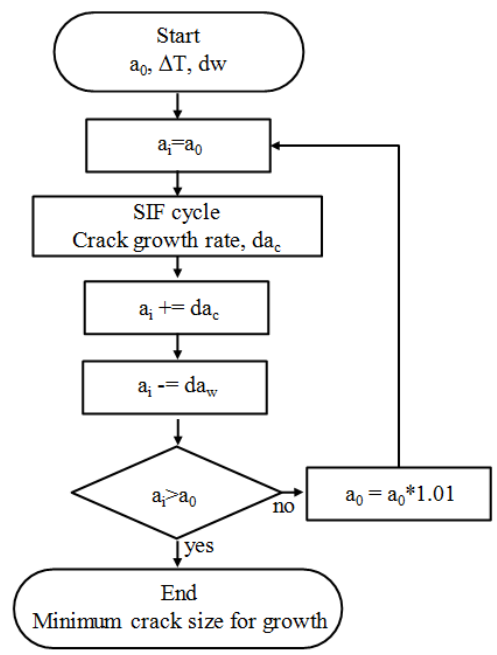

(a)

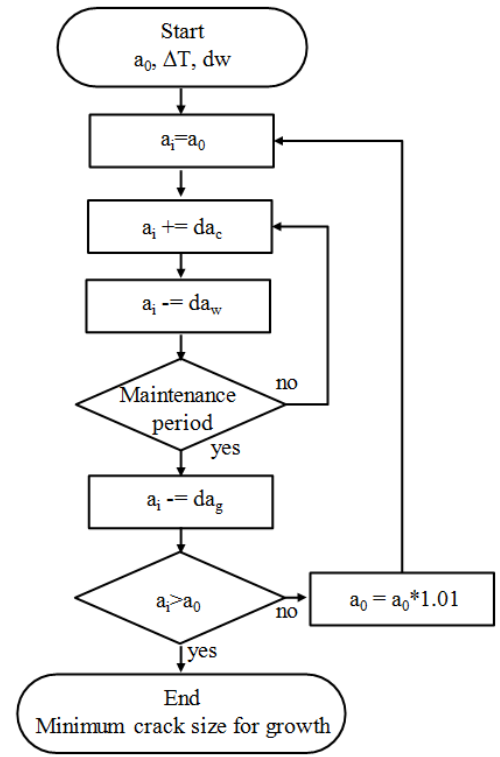

(b)

Figure 4: Flowchart for illustrating how to calculate the MCSG; (a) Periodic grinding is not considered, (b) Periodic grinding is considered.

\subsection{Dynamic force measurement}

To consider a dynamic load increment, when a train runs on a rough rail surface in high speed, we measured the wheel load during a train running on a high speed rail in Korea. The load increment will be larger if there is a crack, because the crack will bring irregularity in rail surface. For this purpose, we made an 
instrument wheel-set as shown in fig. 5(a) and measured vertical and lateral load at every $90^{\circ}$ rotation of wheel. The train ran in tangent line with the speed from 210 to $303 \mathrm{~km} / \mathrm{h}$. Fig. 5(b) is the change in wheel load during the running. They are pointed with the speed. We can see the distribution of wheel load increases with the speed. The maximum load increment is measured as $96.7 \mathrm{kN}$ at $302 \mathrm{~km} / \mathrm{h}$ and it is about $67 \%$ increment of its wheel load in static state. This means that the maximum $96.7 \mathrm{kN}$ can be applied at right above the crack.

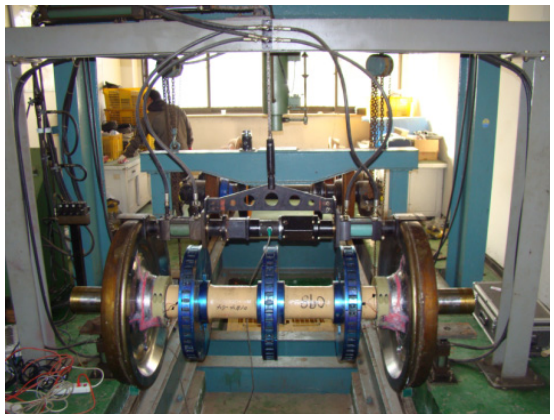

(a)

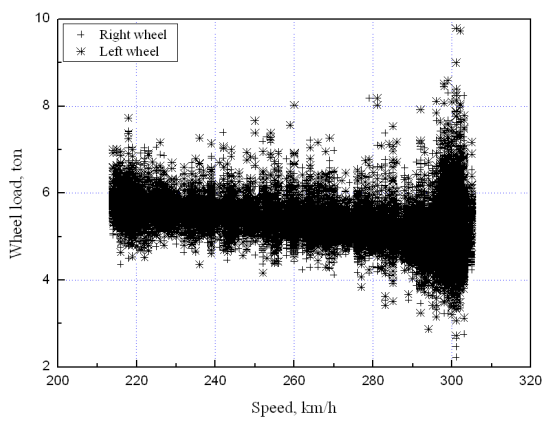

(b)

Figure 5: The instrument wheel-set and the change in wheel load versus the train speed increment; (a) Instrument wheel-set; (b) Wheel load variation in high speed tangent line.

\subsection{Contact patch size}

To apply the Hertzian contact theory, the contact area between wheel and rail should be determined. The high speed train in Korea uses a $920 \mathrm{~mm}$ diameter wheel and the UIC60 rail. The gradient of wheel thread is 1/40 and the rail is $1 / 20$. To calculate the contact patch size, we performed finite element analysis. Fig. 6(a) shows the finite element analysis model for wheel rail contact analysis. The contact region mesh consisted of the minimum $1 \mathrm{~mm}$ size of elements to increase the accuracy in the contact region and the other wheel region mesh consisted of much bigger elements to reduce the analysis time. The reduced 8 node brick element was used to construct the model. The Young's modulus $(E=207 \mathrm{GPa})$ and the Poisson's ratio $(v=0.3)$ were used in the both wheel and rail model, respectively.

The load was applied at the center of wheel where the wheel and the axle were press fitted and the surface friction coefficient between wheel and rail was considered as 0.3. Fig. 6(b) shows the distribution of contact pressure and the size of contact patch. In the static loading case, the maximum contact pressure was calculated as $1,167 \mathrm{MPa}$ and the longer axis of contact ellipse $\left(2 \mathrm{c}_{\mathrm{x}}\right)$ was $14.42 \mathrm{~mm}$ and the shorter axis of contact ellipse $\left(2 \mathrm{c}_{\mathrm{y}}\right)$ was $11.36 \mathrm{~mm}$. In the dynamic loading case, the maximum contact pressure is calculated as $1,593 \mathrm{MPa}$ and the longer axis of contact ellipse is $20.00 \mathrm{~mm}$ and the shorter axis of contact ellipse is $13.20 \mathrm{~mm}$. 


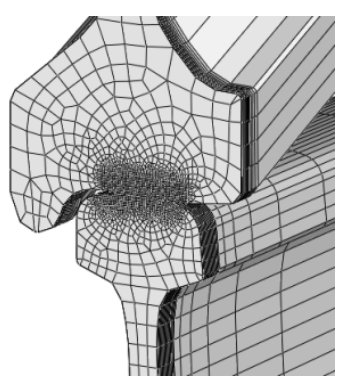

(a)

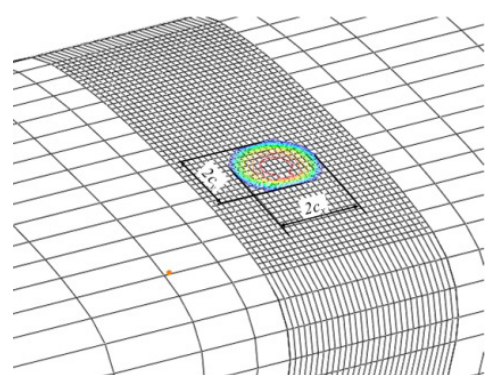

(b)

Figure 6: Finite element analysis model for wheel/rail contact and calculated contact patch size.

\section{Minimum crack size for growth}

\subsection{Wear effect}

The MCSG and crack growth rate about an inclined semi-circular crack under rolling contact were calculated. The angle between the crack and the normal to the edge was $60^{\circ}$. Two maximum contact pressure $\left(\mathrm{p}_{0}=1174,1593 \mathrm{MPa}\right)$ cases were considered and the relevant contact patch sizes were applied respectively. The surface friction coefficient is known as about 0.45 in a dry condition, about 0.2 in a wet condition and 0.01 in an oily condition. So we consider the surface

Table 1: Input condition for parametric analysis for a semi-circular crack under an elliptical Hertzian contact.

\begin{tabular}{|c|c|c|c|c|c|}
\hline Case & $\begin{array}{c}\mathrm{p}_{\mathrm{o}} \\
\mathrm{MPa}\end{array}$ & $\begin{array}{c}\mathrm{c}_{\mathrm{x}} \\
\mathrm{mm}\end{array}$ & $\begin{array}{c}\mathrm{c}_{\mathrm{y}} \\
\mathrm{mm}\end{array}$ & $\mu_{\mathrm{s}}$ & $\begin{array}{c}\mathrm{da}_{\mathrm{w}} \\
(\mathrm{nm} / \text { cycle })\end{array}$ \\
\hline 1 & \multirow{12}{*}{1174} & \multirow{12}{*}{7.21} & \multirow{12}{*}{5.68} & \multirow{3}{*}{0.10} & 0.50 \\
\hline 2 & & & & & 1.00 \\
\hline 3 & & & & & 1.50 \\
\hline 4 & & & & \multirow{3}{*}{0.20} & 0.50 \\
\hline 5 & & & & & 1.00 \\
\hline 6 & & & & & 1.50 \\
\hline 7 & & & & \multirow{3}{*}{0.30} & 0.50 \\
\hline 8 & & & & & 1.00 \\
\hline 9 & & & & & 1.50 \\
\hline 10 & & & & \multirow{3}{*}{0.40} & 0.50 \\
\hline 11 & & & & & 1.00 \\
\hline 12 & & & & & 1.50 \\
\hline 13 & \multirow{3}{*}{1593} & \multirow{3}{*}{10.00} & \multirow{3}{*}{6.51} & \multirow{3}{*}{0.20} & 0.50 \\
\hline 14 & & & & & 1.00 \\
\hline 15 & & & & & 1.50 \\
\hline
\end{tabular}


friction coefficients as $\mu_{\mathrm{s}}=0.1,0.2,0.3$ and 0.4 . The crack face friction coefficient was considered as the same value of surface friction coefficient because the crack mouth was opened to the surface of rail, and the lubrication seepage was expected. Although the wear rate changed with various contact conditions, we considered it as constant $\left(\mathrm{da}_{\mathrm{w}}=0.5,1.0\right.$ and $1.5 \mathrm{~nm} /$ cycle $)$ for the simplicity. The difference between rail neutral and operating temperature was assumed as $20^{\circ} \mathrm{C}$. Total of 15 modelling cases for a semi-circular crack were calculated. Details of modelling run are shown in table 1.

Fig. 7 shows the change in calculated MCSG with the change of wear rate and the maximum Hertzian contact pressure, respectively. Fig. 7(a) shows the MCSG against wear rate. It can be seen that the crack size increases with the wear rate, because the higher wear rate truncates more crack mouth it means that the crack is more difficult to grow to the crack size which the continued crack

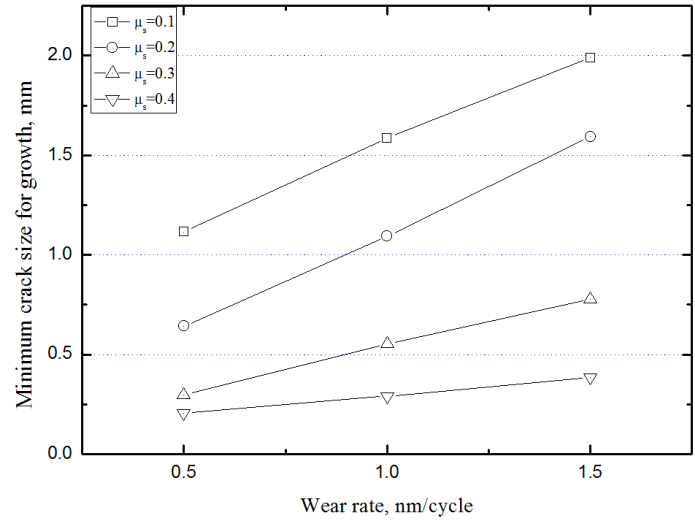

(a)

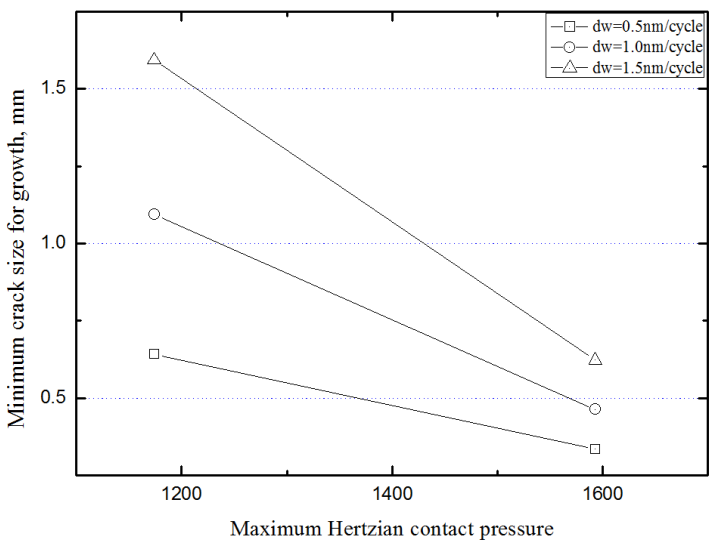

(b)

Figure 7: Comparison of the MCSG varying the dominant contact parameter (a) Surface friction; (b) Maximum contact pressure. 
growth is started. While the crack size decreases with increasing the surface friction coefficient. It is because the high surface traction applies more stresses at the crack tip. The MCSGs are varied from 0.207 to $1.989 \mathrm{~mm}$ depending on the contact conditions. Fig. 7(b) shows the MCSG against the maximum Hertzian contact pressure. It can be seen that the minimum crack size decreases fast as the contact pressure increases.

Fig. 8 shows the change in predicted crack growth rate with the change of surface friction coefficient and the maximum Hertzian contact pressure, respectively. The initial crack size to start simulation was determined as the longest minimum crack size among those in the same contact condition which were calculated previously. Fig. 8(a) shows the crack growth rate versus crack size under the conditions; $\mathrm{p}_{0}=1174 \mathrm{MPa}, \mathrm{da}_{\mathrm{w}}=0.5 \mathrm{~nm} /$ cycle. In this case, the initial crack size was determined as $1.183 \mathrm{~mm}$. In case of low friction coefficient, the traction is expected to be low, therefore slow crack growth rate is acquired. But it also brings low crack face friction which stops crack growth in sliding mode. So the crack growth rate is fast increased when the crack size is over a certain size.

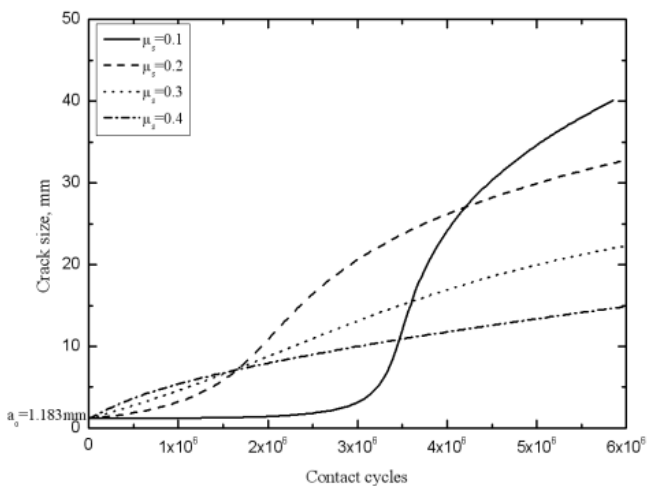

(a)

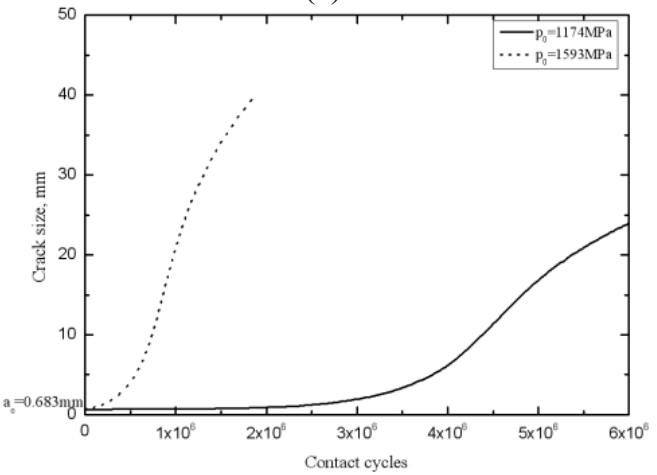

(b)

Figure 8: Comparison of crack growth rate with the minimum crack size as initial crack size; (a) Traction coefficient; (b) Maximum contact pressure. 
In case of high friction coefficient, high traction and high crack face friction are expected and a stable crack growth is acquired. Fig. 8(b) shows the maximum contact pressure effect on the crack growth rate under the condition; $\mu_{\mathrm{s}}=0.2$, $\mathrm{da}_{\mathrm{w}}=0.5 \mathrm{~nm} /$ cycle. In this case, the initial crack size was determined as $0.685 \mathrm{~mm}$. It can be seen from the graph that the crack growth rate is highly relying on the increment of the contact pressure. The crack growth is too fast with $\mathrm{p}_{0}=1593$ $\mathrm{MPa}$ and it seems that it will not be happen in real case.

\subsection{Periodic grinding effect}

The periodic grinding effect was analyzed by implementing a hypothetical example in a railway line. A value of $0.3 \mathrm{~mm}$ was assumed for the grinding depth of rail surface once every 10 MGT based on the general periodic grinding scheme. For the realistic simulation, the current high speed railway operating condition in Korea was implemented to calculate the grinding interval. Since one sets of the high speed train consists of total 20 vehicles and 46 wheel sets, total 391 tons $(46 \times 8.5$ tons $=391$ tons $)$ would be accumulated when the train-set passes over a crack. And the train-set operated average 63 times a day in a single track. Therefore total accumulated gross tons per a day would be $63 \times 391$ tons $=$ 24,633 tons. Normally around every 406 days, it reached the 10 million gross ton. The 15 modelling cases were re-calculated by considering grinding interval. Fig. 9 shows the calculation results. We cannot see much difference in the MCSG in this analysis method. It is because we only consider the phase II crack growth, but the period grinding gives much effect on crack initiation. But we find that once a crack grows over the MCSG then the growth rate is much higher than periodic grinding rate.

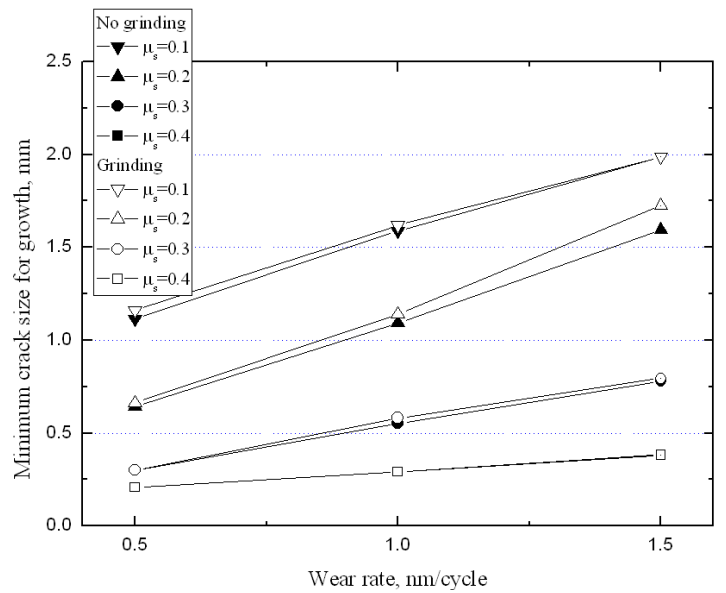

Figure 9: Comparison of the minimum crack sizes for growth when the periodic grinding is considered, semi-circular crack, the temperature difference between the neutral and operating is considered as $20^{\circ} \mathrm{C}$. 


\section{Conclusion}

In this study, the minimum crack sizes for growth and crack growth rates were calculated based on the "2.5D" model which was suggested by Fletcher and Kapoor. For this purpose, computer software named "RailDoctor" has been developed by implementing " $2.5 \mathrm{D}$ " model and verified it by comparing the SIF results with those of well known solutions. Parameter analyses were performed by varying dominant contact parameters such as, contact pressure, surface friction coefficient, wear rate. Based on the analysis results we can conclude that;

(1) The minimum crack sizes for growth were varied from 0.207 to $1.989 \mathrm{~mm}$ depending on the contact conditions.

(2) The contact pressure gives the most significant effect on the minimum crack size for growth. The minimum crack size for growth is longer as the maximum contact pressure is decreasing.

(3) The crack size is longer as the surface traction coefficient is decreasing. While the crack size is shorter as the wear rate is decreasing.

(4) Once a crack grows over the MCSG, then the periodic grinding does not give much effect for stopping the crack growth.

\section{References}

[1] Fischer, F.D., Daves, W., Pippan, R. and Pointner, P., Some comments on the surface crack in rails, Fatigue Fract Engng Mater Struct, 2006;29:938948.

[2] Evans, G., Fletcher, D.I., Franklin, F. and Kapoor, A., Management and understanding of rolling contact fatigue - Phase 2 crack growth, Rail Safety and Standard Board, 2007.

[3] UIC 725, Treatment of rail defect, International Union of Railway, 2007.

[4] Carroll, $\mathrm{R}$ et al, Recommendation of and scientific basis for minimum action rules and maintenance limits, INNOTRACK Deliverable report D4.2.6, 2006.

[5] Bogdanski, S., Olzak, M. and Stupnicki, J. Numerical modelling of a 3D rail RCF 'SQUAT'-type crack under operating load. Fatigue \& Fracture of Engineering Materials \& Structures, 1998;21: 923-935.

[6] Bogdanski, S. and Brown, M. W. Modeling the three-dimensional behavior of shallow rolling contact fatigue cracks in rails. Wear, 2002; 253:17-25.

[7] Bogdanski, S., Lewicki, P. 3D model of liquid entrapment mechanism for rolling contact fatigue cracks in rails. Wear, 2008;265:1356-1362.

[8] Kaneta, M., Murakami, Y. and Yatsuzuka, H., Analysis of surface crack propagation in rolling line contact, ASLE Trans., 1985; 28: 60-68.

[9] Kaneta, M., Suetsugu, M., Murakami, Y., (1986) Mechanism of surface crack growth in lubricated rolling/sliding spherical contact. ASME Journal of Applied Mechanics, 1985; 53: 354-360. 
[10] Kaneta, M., Murakami, Y., Propagation of semi-elliptical surface cracks in lubricated rolling/sliding elliptical contacts. ASME Journal of Tribology, 1991;113: 270-275.

[11] Rooke, D.P., Rayaprolu, D.B., and Aliabadi, M.H., Crack line and edge green's functions for stress intensity factors of inclined edge cracks. Fatigue and Fracture of Engineering Materials and Structures, 1992;15: 441-461.

[12] Fletcher, D.I., and Beynon, J.H., A simple method of stress intensity factor calculation for inclined fluid-filled surface-breaking cracks under contact loading, Institution of Mechanical Engineering Part J, Journal of Engineering Tribology, 1999;213:299-304.

[13] Fletcher, D.I., and Beynon, J.H., A simple method of stress intensity factor calculation for inclined surface breaking cracks with crack face friction under contact loading, Proceedings of the Institution of Mechanical Engineering Part J, Journal of Engineering Tribology, 1999;213: 481-486.

[14] Fletcher, D.I. and Kapoor, A., Rapid method of stress intensity factor calculation for semielliptical surface breaking cracks under threedimensional contact loading, To appear in Proceedings of the Institution of Mechanical Engineers Part F, Journal of Rail and Rapid Transit, 2006;220:219-234.

[15] Kapoor, A. and Fletcher, D.I., The effect of residual stress on contact stress driven crack growth in rail-Literature review, Newrail report,WR061106-1, 2006: 13-15.

[16] Engineering Science Data Unit, Contact phenomenon III : Calculation of individual stress components in concentrated elastic contacts under combined normal and tangential loading, HIS, 1994; 85007.

[17] Erdogan, F. and Sih, G.C., On the crack extension in plates under plane loading and transverse shear, ASME J. Basic Eng., 1963;85:519-525.

[18] Bold, P.E., Brown, M.W., and Allen, R.J., Shear mode crack growth and rolling contact fatigue. Wear, 1991; 144:307-317.

[19] Murakami, Y., Stress intensity factor handbook, Pergamon Press, 1987;712-724. 